\title{
Encore Quelques Délocutifs
}

\author{
(Delocutives Again)
}

Rodolfo ILARI

(Departamento de Linguística do Instituto de Estudos da Linguagem da Unicamp)

\begin{abstract}
"On voit pourquoi je voudrais m' opposer à l' utilisation babituelle des concepts de dénotation et de connotation. On s' en sert généralement pour diviser la signification des mots en deux composants distincts dont l'un a trait au référent et l' autre aux valeurs: on juxtapose une description et une évlauation de la réalité. Ce que je retiendrai de Platon c' est au contraire que l'évaluation est antérieure à la description. Je voudrais même aller plus loin et dire que l' aspect évaluatif est l' origine des éventuelles évaluations descriptives données au mot. ${ }^{1}$
\end{abstract}

\begin{abstract}
In this article, I review the concept of delocutive derivation, conceived for the first time by Benveniste, who exemplified it prototypically through the Latin verb salutare (to greet) as a derivation from the noun salus (bealth). I advocate that it might be interesting not to limit this concept to verb derivation and that an enlarged concept of the delocutive process may be useful to explain many derivations in ancient and modern Portuguese. In addition I present some of the problems that the understanding of the delocutive process may bring to a semantics that wishes to consider only the meaning and the reference of linguistic expressions.
\end{abstract}

KEY-WORDs: Delocutives; Meaning; Reference.

\footnotetext{
${ }^{1}$ Oswald Ducrot, «Topoï et sens», in G.Maurand, Lire et enseigner le texte et l' image / Valeurs et cultures [9ème Colloque d' Albi, Langages et Significations] Toulouse,1989., p.3.
}

D.E.L.T.A., 18:EsPECIAL, 2002 (115-129) 
REsumo: Neste artigo, retomo a noção de derivação delocutiva, formulada pela primeira vez por Benveniste, que a exemplificou prototipicamente pela formação do verbo latino salutare ("saudar, cumprimentar") a partir do substantivo salus ("saúde"). Sustento que há interesse em não limitar essa noção à formação de verbos a partir de substantivos, e que uma noção ampliada de delocutividade se aplica a uma série de formações do português antigo e recente. Discuto ainda algumas das complicações que a compreensão do processo delocutivo traz para uma semântica que queira considerar apenas o sentido e a referência das expressões.

Palavras-Chave: Delocutivos; Sentido; Referencia.

Quand Benveniste a défini la dérivation délocutive, l' idée qu' il faut nécessairement avoir recours à la situation où les mots sont utilisés pour expliquer d' une façon vraisemblable l' évolution de leur sens était déjà bien établie en linguistique. Cette idée avait même été adoptée comme principe par plusieurs écoles linguistiques qui s'étaient consacrées à l'étude de l'évolution du sens au lieu de reconstituer et comparer l' évolution des formes dans l' esprit de la linguistique néo-grammairienne.

L' importance accordée aux situations où intervient le langage pour que la signification puisse être expliquée d'une façon adéquate est bien visible, par exemple, dans le titre de la revue Wörter und Sachen, fondée au début du vingtième siècle. Le rapprochement qui y est fait entre les «mots» et les «choses» est en lui-même tout un programme: on n'arrivera pas à comprendre ce qui s' est passé dans l' histoire des mots si on renonce à les étudier en rapport avec les choses, c' est a dire, avec les objets qu' ils nomment dans les différentes situations d'emploi.

Ce programme a tout d' abord amené à expliquer pourquoi il s' avère si souvent qu' un état plus ancien de la langue présente une seule forme pour telle ou telle signification, là où un état plus récent de la même langue présente une pluralité de formes: pourquoi, par exemple, le mot qui nommait la charrue en latin a été remplacé par toute une série de formes dans les langues romanes: cette diversité s' explique quand on se rend compte que les différents outils employés dans le domaine roman pour labourer la terre ne sont pas les mêmes au delà de leur fonction commune: autrement dit, les choses qu' on serait tenté de considérer comme les signifiés d' un même signifiant n' ont pas la même apparance, sont fabriquées par 
des processus particuliers et s' associent à des techniques plus ou moins locales. Qu' il s'agisse là du «même objet» c' est une illusion, dûe en grande partie au fait qu'on continue a chercher les aboutissants de l' évolution phonétique d' une même forme.

La décision de considérer les situations d'emploi avait permis qu' on propose une solution pour un certain nombre de mystères de l' histoire des langues. En particulier, elle avait permis d' expliquer pourquoi à un moment donné une dérivation phonétique s' estompe et une autre commence, un mot donnant le relais à un autre avec lequel il n'a aucun rapport évident de forme ou de sens à première vue. Dans le domaine des langues romanes, un des cas les plus célèbres de cette situation est, comme chacun sait, celui des mots qui désignent le foie. Le mot pour le foie était en latin iecur, gén. ieconis, apparenté au grec hepar, qui est à l' origine de formations savantes comme hépatite, hépatique, et de formations semi-savants comme le nom du remède brésilien hepatovis qui à en croire à la publicité serait un puissant antidote de la feijoada. Il n' est pas question, bien sûr, de vouloir rattacher les formes foie, fígado, etc. a iecur, mais la disparition de cette dernière forme avait posé aux romanistes un problème qui n’a commancé a se résoudre qu' au moment où l' on a pu constater qu' une des techniques les plus efficaces pour obtenir des foies gras consistait à gaver les oies avec des figues: une oie engraissée avec des figues avait un iecur ficatus: c'est, bien sûr, l'adjectif (non pas le substantif) de ce syntagme nominal qui a donné foie et ses cognats romans. Une explication pareille a été proposée pour les mots qui désignent le lard en portugais et en espagnol: d' après cette explication, le mot portugais toucinho, qui n' a rien à voir, phonétiquement, avec son équivalent latin crassus, se rattacherait à taxuninus, formé à son tour sur taxus (le petit animal omnivore qu' on appelle en français blaireau): à un moment donné de l' histoire du roman, dans la Romania Occidentale, les gens d' Ibérie auraient parlé de n' importe quel lard comme si c'était du lard de blaireau et là encore l'adjectif l'aurait emportée sur le substantif.

L' idée d' une «dérivation délocutive», que Benveniste a lancée dans un article de $1958,{ }^{2}$ apporte, elle aussi, la solution d' un certain nombre de problèmes d' étymologie en se fondant sur la situation d' emploi des expressions, mais elle est d' une nature assez différente. Reprenons son exemple le plus célebre, qui concerne les mots latins salus et salutare. Entre

\footnotetext{
${ }^{2}$ «Les verbes délocutifs», repris dans Problèmes de linguistique générale, Paris, Gallimard, 1966.
} 
ces deux mots, Benveniste reconnaît sans hésiter un rapport morphologique: c' est sur salus qu' a été formé salutare, mais ce qui est simple du point de vue des formes est loin de l' être du point de vue du sens. Le procédé grammatical qui crée salutare à partir de salus est à première vue celui de la dérivation dénominale, or les verbes formés par dérivation dénominale contiennent normalement l'idée de "faire + adj", donc salutare devrait signifier quelque chose comme "faire sain", "rendre sain”, c' est à dire, "guérir". Pour justifier qu' un sens de salutation ait pu s' imposer, Benveniste fait alors un détour par une situation d' énonciation bien attestée dans le monde romain: celle où les gens, en se rencontrant, ou en s' adressant une lettre, échangeaient des souhaits de bonne santé: Plinius Agricolae suo salutem dicit. C' est à cause du rôle essentiel que le mot salus joue dans ces épisodes énonciatifs, et non pas par sa signification originale que qu' il a pu donner salutare signifiant "saluer". Entre les deux mots il y a plus qu' un rapport de sens dont on pourrait rendre compte par une analyse sémantique immanente: il y a une action linguistique qui est accomplie par les locuteurs, dans laquelle un mot - dans les exemples de Benveniste il s' agit toujours d' un substantif - joue un rôle essentiel.

À bien y réflechir, le phénomène que Benveniste a décrit est assez complexe:

a) il comprend, tout d' abord, de la formation d' un verbe à partir d' un substantif. D' un point de vue strictement morphologique, cela ne pose pas de problèmes.

b) par rapport au sens, il y a au contraire une sorte d' impasse parce qu' un sens nouveau est créé qui n' aurait pu être prévu d' avance à partir du sens courant du substantif;

c) l' impasse disparaît quand on réussit à démontrer que le nom en question intervenait dans des actions verbales d' un type spécifique ...

d) et que, dans la dérivation, il a été, pour ainsi dire retenu comme représentant de l' action verbale prise dans son ensemble.

Les actions verbales que les locuteurs accomplissent dans leur activité langagière sont trés nombreuses et très diversifiées, dans n' importe quelle langue humaine. Benveniste a donc raison de s'attendre à ce que la dérivation délocutive joue un rôle important dans l' évolution de toutes les langues humaines. Je pense qu' il a raison aussi de signaler que parfois ce processus de dérivation délocutive n' arrive pas a se compléter. Mais il y a 
une certaine contradiction entre cette dernière constatation et le fait que Benveniste préfère parler de «verbes délocutifs». En effet, cette insistance à parler de verbes délocutifs plutôt que de dérivation délocutive restreint la portée de l' explication envisagée par Benveniste.

Il y a quelques années, dans un article dont je reprendrai quelques exemples par la suite, $\mathrm{j}$ ' ai essaié de convaincre mês amis du GEL (le «Grupo de Estudos Lingüísticos do Estado de São Paulo») qu' on a intérêt à retenir l' idée de dérivation délocutive pour expliquer un certain nombre de faits plus ou moins récénts de l' histoire du portugais. Si l' on s' en tient à la formation de verbes, l' inventaire de faits qu' on dresse est au demeurant assez mince. Nous trouvons, bien sûr esmolar, qui a un rapport morphologique évident avec esmola. Ces deux mots sont les développements portugais d' une voix chrétienne eleemosyna, dérivée d' une la racine grecque (elêemôemon = "pitoyable»), qui se rattache à son tour à un mot (eleos) qui signifiait, "pitié». Le lien entre le sens original et le sens actuel du mot esmola («pitié» $\mathrm{x}$ "petite quantité d' argent qu' on donne aux pauvres») serait perdu si on ne pouvait se rapporter à cette espèce de rituel langagier où le mendiant fait appel à la pitié de son allocutaire en vue d'obtenir une petite aide en argent. Esmola a donné en portugais le verbe esmolar et l' adjectif esmoler. Seul le premier des deux serait considéré comme un délocutif d' après le critère de Benveniste: il décrit en effet l' action de celui qui demande l' aumône en disant la pitié de son allocutaire; esmoler signifie au contraire «charitable», "généreux envers les pauvres», ce qui renvoie à l' idée de pratiquer la pitié, et non de l' invoquer.

Un autre exemple plus typiquement portugais de verbe délocutif est parabenizar. Ce verbe décrit tout type de félicitations (on peut parabenizar un couple de jeunes mariés, quelqu'un qui vient de conclure une affaire particulièrement avangageuse, quelqu' un qui a passé un examen, quelqu' un qui est guéri d' une maladie...); la formule de félicitations à laquelle ce verbe correspond c' est (meus) parabéns. Encore une fois, l' origine du sens de ce verbe serait perdue si on ne pouvait se rapporter à un rituel verbal où le sens original des mots se confond avec le sens global du rituel lui-même. Pour comprendre l' origine de parabenizar, rappelons nous d' abord que «parabéns» est le pluriel de «parabém». Cette variante au singulier, aujourd' hui très formelle et pratiquement inusitée, est plus transparente en ce qui concerne le sens original de l' expression en question: parabém signifiait exactement ce que signifient les deux mots qui la composent, para bem, c' 
est à dire, pour (votre) bien: le rituel de félicitations tel qu' il existait à l' époque où le mot parabém s' est figé en portugais, probablement influencé par la morale chrétienne, exigeait qu' au lieu de vanter les mérites, la bonne chance ou l' importance des faits de l' allocutaire, on lui souhaite que les faits pour lesquels on le félicite contribuent à la longue à son salut eternel.

Dans le cas de parabenizar, comme dans celui de esmolar on retrouve aisément tous les traits de la description que Benveniste proposait pour salutare: on aboutit a un mot nouveau, ce mot est un verbe, ce verbe est créé sur un nom qui existait préalablement, et le rapport entre les deux serait perdu si on ne faisait pas un «detour par une situation d' énonciation» assez spécifique (dans ce sens assez précis que l' énonciation a dans chaque cas un but très clair: obtenir une aide matérielle, féliciter quelqu' un pour une raison qu' on vient d' apprendre). Mais si ces différents traits son présents, rien ne nous oblige de leur attribuer exactement la même valeur. Ce qui distingue les verbes délocutifs des verbes dénominatifs c' est bien sûr ce que j' ai appelé «détour par l' énonciation». Or, un détour par l' énonciation peut intervenir comme étape essentielle dans la formation de nouveaux sens e de nouveaux mots qui n' appartiennent pas à la classe des verbes. C' est pourquoi au lieu de parler de verbes délocutifs je préfere parler de dérivation délocutive.

Si on jette un coup d' oeil au brésilien familier actuel, on vérifie que que la dérivation délocutive a eté utilisée pour la formation de plusieurs substantifs nouveaux; si ces substantifs ne produisent pas, un verbe à leur tour, cela semble être dû au fait qu' on peut toujours former des locutions verbales à l' aide de ce que les linguistes appellent parfois les «verbes légers». Prenons un exemple. J' arrive du jardin, il a plû, je voudrais rentrer dans la maison et je voudrais éviter que mon chien entre avec moi. J' ouvre la porte, le chien fait comme s' il allait passer à ma droite et passe entre mês jambes. En portugais brésilien très familier, on peut résumer ce qui s' est passé en disant que «O cachorro deu um olé no professor», littéralement, «Le chien a donné (ou fait?) un olé au prof). Le problème qui se pose est, bien sûr, d' expliquer ce mot olé. Pour le faire, il faut se rapporter au football, ce sport où les brésiliens éxcellaient (avant que le roi de France soit chauve et s'apelle Zidane, comme m'a signalé mon ami, le linguiste et footballeur Sírio Possenti). La liste des expressions qui sont passées du football au brésilien commun reste à faire, mais ces expressions sont nombreuses - 
pensons à passar uma rasteira, por tabela, botar um cara de escanteio, bola quadrada, bola murcha, bola no mato... - il n' est donc pas surprenant qu' on retrouve parmi ces expressions quelques cas de dérivation délocutive. Revenons à olé. Les traits les plus remarquables du football brésilien d' autrefois étaient le dribling, les feintes spectaculaires par lesquelles les joueurs d' attaque démoralisaient leurs marqueurs et surtout les passes courtes. Tout brésilien d' un certain âge se rappelle les vieux films où Garrincha feintait son marqueur sans lever les pieds de terre, rien que par le mouvement du corps et de ses jambes crochues. Ce type de feinte, et surtout les passes par lesquelles l' une des équipes garde le ballon et empêche l' équipe adversaire d' y toucher ont un effet démoralisant et suggèrent qu' il ne reste à l' adversaire que le rôle de souffre-douleur. Ce n' est pas étonnant que ces situations aient évoqué aux brésiliens la tauromachie, et surtout l' habitude de crier «olé» quand le torero dirige les mouvements du taureau avec les mouvements de sa cape. Voilà établie l' origine probable du mot olé. Le «détour par l' énonciation» a permis, dans ce cas, qu' une exclamation devienne un substantif commun, plus exactement un substantif «comptable». Si, par la suite, la langue n’est pas arrivée a créer un verbe (j’ ai cependant vu «olear» dans une chronique sportive récente que je n' ai pas été capable de récupérer par la suite), c' est parce que les verbes dar, levar, et éventuellement d' autres «verbes légers» permettent de préciser sans équivoque si le sujet de la phrase est l' agent ou le patient du «olé». C' est le paradigme qu' on retrouve par exemple dans

«O cachorro deu um olé no professor» $=$ «O professor levou um olé do cachorro»

«O assaltante deu um tapa no professor» $=$ «O professor levou um tapa do assaltante»

«A recepcionista deu um beijo no prefeito» $=$ «O prefeito ganhou um beijo da recepcionista»

etc.

Le type de dérivation, où un mot maintient sa forme originale mais change de catégorie grammaticale (ce n' est pas la peine que nous nous attardions ici sur le changement d' ouverture e $>$ e entre l' espagnol et le portugais) a reçu des grammairiens le nom de «dérivation impropre», et c' est surtout à des résultats de ce genre qu' aboutit aujourd' hui la dérivation délocutive du brésilien familier. Comme il fallait s'y attendre, toute une énonciation et toute une situation bien précise sont évoquées dans la 
nouvelle création lexicale, de sorte que quelquechose de la situation qui a déclenché tout le processus est gardé - en général un type particulier de rapport entre les interlocuteurs. Em voici quelques autres exemples:

1) Des vêtements qui attirent l' attention par des couleurs scandaleuses ou par un modèle inusité peuvent être décrits comme «umas roupas (bem) cheguei» (littéralement, «des vêtements (assez) je suis arrivé(e)» / (assez) me voilà»). La même expression peut être appliquée aux personnes qui les portent (uma dona muito cheguei, litt. «une bonne femme très je suis arrivé(e)»). Le verbe chegar, à la première personne du singulier du passé simple est devenu un adjectif (à ce point qu' on peut l' utiliser au superlatif). Pour cela, il a fallu que «cheguei» soit entendu comme une partie d' un acte d'énonciation où quelqu' un attire l' attention sur as personne («me voilà», «ne manquez pas de me voir», etc.)

2) Malgré ses connotations vulgaires et son histoire machiste (ou peutêtre grâce à elles) l' expression dá ou desce est très efficace pour caractériser une action verbale qui amène à sortir d' une impasse. On peut donc avoir, même à l'université, des dialogues comme «

A - O próximo número de nossa revista vai sair atrasado por causa do Rodolfo que não entrega seu artigo sobre os delocutivos

B - Deixa comigo. É já que ligo para ele e dou aquele dá ou desce"

(Littéralement:

A - Le prochain numéro de notre revue finira par sortir avec du retard à cause de Rodolfo, qui ne remet jamais son article sur les délocutifs. /

B - Je m' en occupe. Je vais l' appeler tout de suite et je vais lui donner un bon "donne ou descend").

La scène évoquée par cette expression se passe idéalement dans la cabine d'un camion, entre une femme et un camionneur impatient; c' est le camionneur qui parle pour annoncer à la femme que, si elle ne passe pas tout de suite à l' acte sexuel, elle doit quitter les lieux. Le substantif dá-oudesce (qui n' apparaît pas, bien sûr, dans les dictionnaires) garde quelquechose de la situation énonciative originale. Par exemple, la personne qui prend l' initiative du dá ou desce (c' est à dire, la personne qui a dans l' histoire le rôle d' agent) doit avoir quelque autorité sur la personne à qui le 
dá ou desce est addressé; si on rapporte une conversation comme un "dá ou desce", on peut s' attendre à ce que les rapports entre les interlocuteurs ne soient pas très amicaux, etc.

3) On peut imaginer des histoires délocutives pour chega pra lá, passa moleque et não me toques ("O fulano está botando as asinhas de fora. Precisa levar urgentemente um chega pra lá", "Fulano de tal pensa que me assusta com um passa moleque", "Maria anda cheia de não me toques"). Dans tous ces cas, on part d' une énonciation dont on garde essentiellement la fonction. Ainsi, chega pra lá et passa moleque désignent des dialogues où il s' agit de remettre quelqu"un à sa place, et "cheio de não me toques" caractérise une personne grognonne, qui abuse du droit de se plaindre... Dans tous ces cas, je pense que nous avons des délocutifs à part entière, qu' on doit sans doute distinguer de "pseudo-délocutifs" qui retiennent seulement quelques caractéristiques très superficielles de l'énonciation originale, tels que nhenhenbém, patati patata / tititi, blablabla qui renvoient à une conversation dont ils ne retiennent qu' une vague impression sonore...)

Avant de terminer la partie descriptive de cet exposé, je voudrais citer trois cas qui sont partiellement différents de tous ceux que j'ai décrits jusqu' à ce point et qui nous permettrons d' élargir tant soit peu la portée des questions qui seront soulevées dans la partie "théorique" qui va suivre.

Le premier de ces cas a été découvert par la linguiste brésilienne Patrícia Rodrigues, à l' époque où elle fesait ses recherches de doctorat à l' Université de Montréal. Il concerne une des dénominations par lesquelles les touristes provenant d' Argentine sont connus dans les états du sud du Brésil (j' allais dire le midi du Brésil): damedos. La finale -s de ce mot ne doit pas nous tromper: il ne s' agit pas du pluriel d' un mot portugais; mais d' une phrase espagnole composée de l' impératif $d a$, plus le pronom me plus le numéral dos. Donc, sa traduction littérale en français serait quelque chose comme "donnez m' en deux". Pour expliquer que cette expression ait fini par identifier le touriste argentin, il faut se rapporter à l' histoire très accidentée des échanges commerciales entre le Brésil et l' Argentine, qui a eté marquée, au cours des dernières decennies, par des oscillations extrêmes, tout à fait impensables pour un éuropéen. Ce qui compte ici c' est que le tourisme entre ces deux pays n' a jamais connu de réciprocité: les Brésiliens envahissent Buenos Aires aux époques où le peso se dévalorise: ils sont alors identifiés par l' expression baratíssimo, par laquelle 
ils réagissent aux prix des articles argentins même quand ils ne sont pas acheteurs; à leur tour les argentins envahissent les plages du sud du Brésil quand la monnaie brésilienne perd son pouvoir d'achat. À ce moment-là se produit la scène dont rêvent les commerçants brésiliens: les familles argentines entrent dans leurs établissements, se font montrer tout genre de marchandises et, séduites par des prix qu' elles considèrent trop avantageux pour ne pas saisir l' occasion, achètent deux pièces de n' importe quoi. Dá-me dos.

Il n' est pas étonnant que [damedos] soit devenu une manière pratique de se réferer aux argentins quand on les représente comme des touristes. Le mécanisme qui a déclenché cette formation est bien évident: on retient une expression capable d' évoquer une situation stéreotypée, et on s'en sert pour dénominer le personnage qui est censé la prononcer dans cette situation. C' est, on le voit, le même mécanisme qui a fait que, dans mon université, les enseignants provenant d' autres pays d' Amérique latine où l' espagnol est la langue maternelle, soient parfois identifiés comme des "pero-que-s": ces enseignants sont alors représentés comme des intellectuels qui argumentent beaucoup, mais qui n’ arrivent pas à s' exprimer dans un portugais impeccable - la preuve en serait précisément la difficulté de remplacer pero que par son équivalent portugais, l' inexpressif porque.

Le cas signalé par Mlle. Rodrigues damedos, a ce mérite: il attire notre attention sur le fait que les étrangers (ou, plus précisément, les gens qui parlent leur langue à l' étranger) sont les victimes potentielles d' une dérivation délocutive. Pensons à quelques caractérisations des italiens (pour des raisons évidentes j'en sais un peu plus sur les italiens que sur d'autres peuples): au Brésil, nous trouvons les expressions carcamano et buona gente; en France, macaroni, etc. : il s' agit, dans toutes ces langues, d' expressions qui ont été reprises de l' italien lui-même. Une dérivation délocutive, si elle n' est pas évidente, reste cependant possible (les italiens seraient ceux qui en parlant d' eux mêmes insistent à se traiter de "buona gente"; les italiens seraient ceux qu' il faut engueuler en les traitant de carca-mano, parce qu'ils volent sur le poids des marchandises en appuyant de la main sur le plat de la balance. Le mot qui est passé à l' allemand désigne au début le manoeuvre qui travaille comme aide-maçon, qu' on appelait par cette désignation. C' était en effet le rôle professionnel qu' on réservait dans l' industrie du bâtimant aux immigrants italiens nouveu-arrivés. Remarquons en passant que dans tous ces cas quelque chose de la 
phonétique ou de la phonologie de la langue originelle (ici, l' italien) passe dans l' autre langue, que ce soit l'affriquée de gente, le a non nasalisé de carcamano, ou le $-i$, marque du pluriel en italien, Il va sans dire que tous ces traits phonétiques jouent un rôle connotatif très fort - par le même principe qui fait que tout propriétaire de restaurant italien se sent autorisé à multiplier les -is et les consonnes doubles (d'où les créations saugrenues qui apparaissent das les lists de mets - telles "calzoni ai vongoli” ou "penni all' arrabbiatti”). Au Brésil, qui a acueilli tant d' immigrants des plus différentes origines, une analyse sérieuse des dénominations qui ont été appliquées à ces immigrants, et de l' importance qui y a joué la dérivation délocutive mériterait sans doute d' être faite: brimo, gaidjin...

Mon deuxième cas concerne un des noms par lesquels a été connu vers les années mille neuf cent quatre vingt le modèle le plus célèbre de voiture fabriqué par Volkswagen, la Sédan 1200. Partout ailleurs les noms de ce modèle évoquent un type d' insecte (d' où des appellations comme coccinelle, bug, maggiolino...). Les brésiliens ont appelé tout d' abord ce modele de voiture "fusca", "fusquinha", une appellation qui dérive phonétiquement de Volks et qui a été receuillie par les dictionnaires récénts du brésilien (la dernière édition du Dicionário Aurélio fait même une distinction nette entre fusca e fuscão, ce dernier modèle se caractérisant par une cylindrée de 1500 cc). Le fusca a été la voiture la plus populaire du Brésil pendant une vingtaine d' années, mais vers 1980 son prestige était déshormais en baisse: on commença alors à lui reprocher d' être né d' un vieux projet militaire (d' òu le circuit de refroidissement à l' air), et on l' accusa d' offrir peu de comfort, peu de visibilité et peu de luxe. À ce moment-là les brésiliens ont invente pour ce modele de voiture qui tombait en disgrâce un autre nom: pois é. Pourquoi pois é? Pois é fait partie de la grande famille d' expressions (comme sim, isso mesmo, correto, exato, acertou, falou, falou e disse, pode escrever ...) qui marquent l' assentiment. Ce qui le caractérise par rapport aux autres expressions de cette famille c' est qu' il ajoute à l' idée de confirmation l'idée que ce qu' on confirme est en même temps à plaindre. Quelque chose comme "malheureusement oui", "bah oui!” ou “c' est ça, même si ce n' est pas fameux”. On peut imaginer le chemin par lequel pois é devient le nom d' une voiture autrefois noble et maintenant déchue de son trône dans l' imagination des brésiliens: A, qui rentre chez lui en voiture offre á $\mathrm{B}$ de le déposer quelque part et $\mathrm{B}$ accepte. B n' a jamais vu la voiture de A. Quand ils arrivent au parking, B voit la 
voiture de A et pose la question embarassante: "Você tem um fusca"? A répond: “Pois é”. La réponse n' aurait pas été la mème, vers les années ' 80 si la voiture avait été une Ford, une Chevrolet ou une Mercedes: mais à 1' époque on utilisait déjà pois é pour s'excuser du fait que la voiture n' était pas plus confortable. Peu à peu, pois é est devenu un synonyme (péjoratif) pour fusca, donc un substantif comptable: à un certain moment, vers la fin des années 1980, on pouvait dire en portugais du Brésil "tenho um pois é”, "o único carro da casa é um pois é”, "como a estrada da chácara é ruim, só vou no pois é". Bien que son idéntification avec une époque heureuse et optimiste de l' économie brésilienne lui ait accordé une période de survie lors du mandat du président Itamar Franco (1992-1994) les jours du fusca / pois é étaient deshormais comptés. Il y a des pois és qui roulent encore, mais ce modèle ne se fabrique plus. Mas la dérivation qui a fait circuler pendant vingt ans l' expression pois é comme dénomination d' une voiture populaire n' en est pas moins un fait linguistique remarquable.

Je termine cet inventaire de cas en résumant les conclusions auxquelles je suis arrivé, dans un autre travail, à propos de l' expression interrogative "falou?". On s' attend à rencontrer cette expression dans des contextes comme
A - No churrasco de amanhã, você compra a carne, falou?
B - Falou. Eu levo a carne.

La phrase de A pourrait être traduite par quelquechose comme "Pour le barbecue de demain tu achètes la viande, d' accord?”; A énonce donc sa phrase avec l' intention d' obtenir une confirmation d' accord de la part de B. Ce qui fait problème dans cette phrase c' est que A utilize le verbe falar ("parler", "dire") à la deuxième personne du singulier (você = tu) en lui appliquant le ton interrogatif, ce qui devrait produire pour l' ensemble de la phrase un sens comme "Pour le barbecue de demains soir tu t'occupes de la viande, as-tu dit"? Mas si A avait voulu exprimer ce sens il aurait dit autre chose:

$A^{\prime}$ - No churrasco de amanhã você compra a carne (foi isso que) você falou?

A" - No churrasco de amanhã você leva a carne, é isso? 
Pour expliquer ce qui se passe dans la question posée par A, il vaut donc mieux partir du sens que $B$ attribue au mot falou dans sa réponse. Ici, falou marque l' assentiment, comme le dixisti de l' Evangile.

\section{Es-tu le fils de l' bomme?}

Tul' as dit.

Dans le brésilien des jeunes gens, il y a eu un moment où falou exprimait le consentiment d' une façon plus expressive que l' adverbe sim, ou la répétition du verbe de la question (A - Você leva a carne? / B - Levo). Apparemment, quand cette façon de marquer l' assentiment était assez bien assimilée, le mot falou a perdu tout contact avec le paradigme verbal d' où il provenait, et il a été analysé en deux parties: un sens propositionnel d'accord, attaché au lexème, plus un sens d'assertion ou d' engagement, attaché au ton affirmatif. Il a suffi d' appliquer au même lexème le ton interrogatif pour que falou? devienne une "demande d'assertion de l' accord". Encore une fois, on ne comprendrait pas ce qui s' est passé si on ne pouvait se référer aux situations dialogiques où le déplacement a eu lieu.

Essayons de faire le point de ce que nous avons vu jusqu' ici. Comme j' ai commenté plusieurs exemples de formation de sens en portugais récent j'ai pu donner l' impression que je voulais faire un peu d' étymologie récente, à titre de variations sur un thème de Benveniste. Comme j' ai dit qu' il vaut mieux parler de dérivation délocutive là où Benveniste parlait de verbes délocutifs, quelqu' un pourrait en conclure que je voulais m' en prendre à Benveniste. J' admets que je n' ai pas été trop clair jusqu' ici sur le but de cet exposé, mais je pense que ceux qui ont bien voulu me suivre devraient tomber d' accord avec moi sur une idée très simple: à savoir qu' il y a dans l' histoire du brésilien un ensemble de faits qu' on arrive à analyser d' une façon plus convaincante si l' on dispose d'une notion plus ou moins tolérante de délocutivité.

Cela dit, ce qui m’ a amené à reprendre mes exemples de dérivation délocutive c' est l' intuition que ce phénomène, aussi marginal qu' il puisse paraître, a quelquechose de fondemental à nous enseigner sur la nature du langage. On a donc intérêt à théoriser un tout petit peu sur la délocutivité, et c' est précisément ce que je compte faire dans les lignes qui me restent. Ce genre de réflexion devrait aussi expliquer pourquoi je pense qu' un 
exposé sur les délocutifs est adéquat dans un colloque consacré à l' indétermination du langage.

Si on reprend les exemples de verbes délocutifs étudiés par Benveniste (tels le verbe latin salutare), et tous les exemples que nous avons trouvés qui s' incluent de façon exacte dans sa définition (tels l' espagnol pordiosar et le portugais esmolar, parabenizar), on constate qu' ils se fondent sur un usage indirect des mots, ou, plus exactement, sur ce que la linguistique anglo-saxonne appellerait volontiers des actes de parole indirects. C' est ainsi que l' allusion à la pitié de l'allocutaire - qui serait à l' origine un constatif dans le classement de Searle - devient un injonctif dans la bouche du mendiant, et que le souhait exprimé par parabéns devient par la suite un commentaire sur une action dont l' interlocuteur a été le protagoniste. Un déplacement analogue pourrait être signalé pour la plupart des exemples que j' ai commentés. L' idée de caractériser la dérivation délocutive en ayant recours à la notion d' acte de parole indirect semble donc juste mais, avant d' y adhérer il faut réflechir sur ce qu' elle presuppose en matière de théorie. Si nous décidons que le mendiant qui dit eleimosiné pour demander une petite somme en argent utilize le mot d' une façon indirecte, c' est, bien sûr, que nous avons décidé qu' il y a des usages directs et des usages indirects, et que nous savons très bien les distinguer les uns des autres. Savons-nous toujours faire ce genre de distinction? Savons nous tracer la ligne qui sépare ce qui est direct de ce qui est indirect?

Dans la perspective des études linguistiques inaugurée par Saussure nous devrions croire que ce genre de distinctions est toujours possible. Mais nous devons nous rappeler que cette perspective est le résultat d' une manoeuvre sur laquelle Saussure a été assez clair et qui a consisté à "se placer d' emblée dans la langue", c'est à dire, dans une systématisation possible des faits de langage. Quand on cherche à faire rentrer dans une systématisation plus rigide des faits comme ceux dont j' ai parlé ici, on a l' impression que les effets de sa manoeuvre sont remis en cause, qu' on se retrouve à un stage de la réflexion linguistique où le concepte courant de système nous cause des embarras au lieu de nous servir d' outil.

Je pense qu' on arriverait au même point si on partait d' autres notions qui ont été créées pour rendre opérationnelles des explications qu' on peut donner à l' intérieur d' un système, telles la notion de sens littéral ou la notion de compositionnalité. Quand on a une dérivation délocutive s' 
accomplit, c' est évident qu' un sens littéral est transgressé, et qu' on ne peut plus calculer la signification de l' énoncé à partir de la signification de ses parties. Mais pourquoi devrions nous entendre que cela fait problème? L' acquisition et la pathologie du langage offrent une quantité de cas où une expression complexe doit être comprise dans son ensemble avant d' être analysée dans ses parties constitutive. Pourquoi devrions nous croire que dans l' usage courant du langage les parties ont une priorité logique sur les épisodes d' usage?

On aura compris, je pense, que la dérivation délocutive nous oblige à remetre en cause l' idée reçue d' unité linguistique, d' une façon beaucoup plus radicale que la plupart des phenomènes diachroniques. C' est un résultat auquel on arrive aussi quand on traite de grammaticalisation, et quand on réfléchit sur la fragilité des classements dont s' est servie la grammaire pendant des siècles. À la limite on devrait se demander "quel genre de science faisons nous?”, mais je ne poserai pas cette question. Je me contente de dire que, parfois, pour comprendre ce qui se passe, on a intéret à renoncer à la clarté des schémas habituels. 